\title{
The Role of Infection and Autoimmunity in Urticaria and Angioedema as a Common Entity
}

\author{
Authors: \\ Michael Rudenko \\ The London Allergy and Immunology Centre, ACARE \& UCARE, London, UK \\ *Correspondence to consultation@UKallergy.com \\ Disclosure: \\ The author has declared no conflicts of interest. \\ Received: \\ 06.06 .20 \\ Accepted: \\ 15.02.21 \\ Keywords: \\ Angioedema, COVID-19, Helicobacter pylori, herpes, urticaria. \\ Citation: \\ EMJ Allergy Immunol. 2021;6[1]:79-85.
}

\section{Abstract}

Chronic spontaneous urticaria with angioedema is prevalent, affecting approximately $1 \%$ of the general population, and has a significant impact on quality of life, according to epidemiological data. This article aims to broaden the view on the mechanisms of urticaria and the role of infection in the current environment. It is not easy to identify the cause of urticaria but appropriate steps to treat an underlying infection can, in some cases, improve the symptoms of urticaria and angioedema, reduce severity and duration, or lead to remission.

Although chronic spontaneous urticaria with angioedema is a multifactorial condition involving inflammation, autoimmunity, and coagulation, IgE-mediated autoimmunity, or autoallergy, is thought to play a major role. Every year, more is learnt about the role of cells releasing mediators, underlying autoimmune processes that lead to the development of mast cell activation and urticaria.

It has become increasingly clear that mast cell roles in immune system responses are not limited to an allergic role; they are key players in protective immune responses, both innate and adaptive, to various pathogens and in defence of some infections.

Several guidelines, consensus papers, and practice parameters have been developed for the management of chronic urticaria. The Dermatology Section of the European Academy of Allergology and Clinical Immunology (EAACl), the Global Allergy and Asthma European Network (GA²LEN), the European Dermatology Forum (EDF), and the World Allergy Organization (WAO) produce a guideline, which is revised every 4 years by a global panel of experts in the field. Infections may be a cause, aggravating factor, or unassociated bystander in chronic urticaria.

The author looked at evidence, using a keyword search, for the role of viral and bacterial infections in acute, acute recurrent, and chronic urticaria and angioedema, including COVID-19, herpes, viral hepatitis, and Helicobacter pylori.

\section{INTRODUCTION}

Urticaria is a highly prevalent, mast-cell-driven skin disorder with recurrence of transient wheals

that can occur with or without angioedema.,2 Urticaria affects between $15 \%$ and $25 \%$ of the population at some point during their lifetimes. ${ }^{3}$ Urticaria is characterised by the development 
of wheals, angioedema, or both. Chronic urticaria is defined by recurrently appearing signs and symptoms for $>6$ weeks. The disease activity of all chronic urticaria subtypes can markedly change over time and differ between individual patients. ${ }^{4}$

The condition tends to be more common in adults than in children and in females than in males, with peak occurrence in the third to fifth decades of life. This condition is marked by the onset of pruritic wheals, which represent wellcircumscribed areas of non-pitting oedema with blanched centres and raised borders that involve only the superficial portions of the dermis and are seen in conjunction with surrounding erythema of the skin. ${ }^{5}$

The discussion of the role of infectious diseases in urticaria has been continuing for more than 100 years. It is evident that the eradication of the infection could lead to the resolution of urticaria; however, a causal relationship with underlying or precipitating infection is difficult to establish. ${ }^{6}$

Different studies have reported that multiple infections range from $37 \%$ to $58 \%$ among patients diagnosed with urticaria. ${ }^{7}$ There are several theories for the pathogenesis of the potential autoimmune nature of this condition, associated with approximately $50 \%$ of cases. ${ }^{8}$ It is understood that the underlying mechanism of urticaria is caused by activation of mast cells and basophils, which release pro-inflammatory mediators that result in increased permeability of blood vessels and irritation of nerve endings, leading to swelling and pruritus. ${ }^{9}$

Current research in chronic spontaneous urticaria (CSU) targets the role of cells and released mediators that lead to the development of urticaria through mast cell activation, with a focus on the underlying autoimmune processes..$^{10}$ Although CSU is a multifactorial condition involving autoimmunity, coagulation, and inflammation," IgE-mediated autoimmunity, or autoallergy, is thought to play a major role. ${ }^{12}$

Histamine release likely occurs through crosslinking receptors by IgG-specific and specific auto-immune IgE autoantibodies derived against antigens, on mast cells and basophils. ${ }^{13}$

IgG anti-FcrR1a induces histamine release irrespective of the degree of IgE sensitisation of the basophils. As proof of concept, histamine release was effectively neutralised in a concentration-dependent manner by preincubating donor basophils with a soluble fragment of FceRla prior to the addition of purified IgG from sera of patients diagnosed with CSU..$^{12}$ It was shown that circulating IgG antibodies against $\operatorname{IgE}$ and the high-affinity $\lg \mathrm{E}$ receptor FceR1 likely played a role. ${ }^{14}$ Approximately 40\% of patients have circulating antibodies to one of these targets, with a higher frequency of positivity in patients who are autologous serum skin test positive. ${ }^{15}$

Anti-FceR1 antibodies are thought to be the more common of the two. FceR1 is found on the surface of both dermal mast cells and basophils, and autoantibodies to this receptor can provoke chronic stimulation and degranulation of these cells in an IgE-independent fashion. ${ }^{16}$ In contrast, IgG anti-IgE antibodies may bind to and cross-link receptor-bound IgE on the surface of mast cells and basophils, leading to activation and degranulation of these cells.

Protein microarray analysis showed that patients diagnosed with CSU have higher IgE levels than healthy individuals, and IgE are directed mainly to thyroid antigens and double-stranded DNA. ${ }^{12}$ Cholinergic urticaria is a frequent form of inducible urticaria, characterised by small, itchy wheals induced by physical activity or passive warming. The underlying causes are not completely understood. Several recent studies have provided evidence that IgE-mediated mast cell activation is of major importance in the pathogenesis. ${ }^{13}$

In approximately $10 \%$ of patients, urticaria is linked to rare factors: allergic urticaria, ${ }^{17}$ salt-dependent aquagenic urticaria that has been reported in adults and two cases have now been reported in children. ${ }^{18}$ Twenty five reports of chronic urticaria and malignancy raised the possibility that chronic urticaria and malignancies are linked in some patients. ${ }^{15}$ Recently, two additional cases of cancer and chronic urticaria have also been reported, with resolution of urticaria once the tumour was removed. ${ }^{19,20}$

Exposure to phthalates (substances used primarily to soften polyvinyl chloride) was shown to increase the risk of acute urticaria in children. ${ }^{21}$ Nearly all of the numerous studies reporting 
evidence for infectious agents triggering acute or recurrent acute urticaria were retrospective observational studies without appropriate controls or were case reports. ${ }^{22}$ There were reports of Mycoplasma pneumoniae infection in $32 \%$ of 65 children with acute urticaria. ${ }^{23}$ There were observations of urticaria symptoms after influenza vaccination. ${ }^{24}$ Upper respiratory or digestive symptoms are common with urticaria associated with infections. ${ }^{25,26}$

Other reports focused on an association between acute urticaria and streptococcal infection, ${ }^{30}$ hepatitis $A^{28}$ and $B$ viruses, ${ }^{30}$ parvovirus B19, cytomegalovirus (CMV), ${ }^{29}$ Coxsackie A9 virus, ${ }^{31}$, enterovirus, influenza $A,{ }^{24}$ and parainfluenza viruses. Viral infection can be a potential trigger and sometimes the main aetiologic agent in causing acute or chronic urticaria. ${ }^{32}$

Several hypotheses exist that hepatitis B or C infection may enhance IgE-induced mediator release from mast cells and basophils. ${ }^{33}$

Protein factor $V$, which is produced during viral hepatitis, can activate human basophils and skin mast cells to release histamine and other mediators. ${ }^{34}$ Factor $\mathrm{V}$ protein acts as an endogenous super-antigen by interacting with the $\mathrm{VH} 3$ domain of IgE to induce the activation of mast cells. ${ }^{35}$

As only $<5 \%$ and $2 \%$ of patients diagnosed with CSU have hepatitis $\mathrm{B}$ and $\mathrm{C}$, respectively, the rates of infection do not appear to be increased in patients diagnosed with CSU, suggesting that viral hepatitis and CSU are not usually linked. ${ }^{36}$

Acute infection with viral pathogens in the Herpesviridae family can trigger acute urticaria, and reactivation of Herpesviridae is associated with cutaneous urticarial-like syndromes. Reactivation of latent Herpesviridae has not been studied systematically in chronic idiopathic urticaria and $\mathrm{CSU}^{37} \mathrm{CSU}$ is an inflammatory disorder with autoimmune features (termed chronic viral urticaria) based on serology, consistent with the hypothesis that reactivation of a latent human herpesvirus (HHV) or viruses may play a role in CSU. ${ }^{37}$

Patients diagnosed with CSU also exhibited serological evidence of increased immune response to HHV-4 (Epstein-Barr virus) but not all patients diagnosed with CSU were infected with Epstein-Barr virus. These observations, combined with case reports of CSU response to antiviral therapy, suggest that HHV-6, possibly interacting with $\mathrm{HHV}-4$ in cutaneous tissues, is a candidate for further prospective study as a co-factor in CSU. ${ }^{37}$

In the beginning of the COVID-19 outbreak in Wuhan, urticaria was self-reported among community-acquired cases in $1.4 \%$ of patients, with an approximately 1:1 ratio of male (50.7\%) and female patients, with an overall median age of 57.0 years. ${ }^{38}$ From a series of 88 patients, $20 \%$ developed cutaneous manifestations including erythematous rash, widespread urticaria, and chickenpox-like vesicles. ${ }^{39}$ In a later publication, cutaneous manifestations of COVID-19 infection included a papulovesicular rash (34.7\%; 25/72), and urticaria (9.7\%; 7/72). ${ }^{40}$

Many bacterial infections have been associated with urticaria manifestation, such as Helicobacter pylori, Streptococcus, Staphylococcus, Mycoplasma pneumoniae, Salmonella, Brucella, Mycobacterium leprae, Borrelia, Chlamydia pneumoniae, and Yersinia enterocolitica. In some cases, the skin manifestations, described as urticaria, could be caused by the presence of the microorganism in the skin, the action of their toxins, or complement activation mediated by circulating immune complexes. Although only a weak association with urticaria of unclear pathogenesis exists, clinicians should consider these bacterial agents in the work-up of the patients diagnosed with urticaria. The eradication of the infection could, in fact, lead to the resolution of urticaria. ${ }^{6}$

Seropositivity of anti-H. pylori antibodies was higher in the urticaria-diagnosed patients than in control groups. H. pylori is a spiral-shaped micro-aerophilic Gram-negative bacterium that colonises the gastric mucosa and induces a strong inflammatory response with release of various bacterial and host-dependent cytotoxic substances. ${ }^{41}$ The existence of a correlation between $H$. pylori and urticaria may help clinicians to find more effective methods to treat patients diagnosed with chronic urticaria. ${ }^{32}$

H. pylori is a risk factor for developing chronic urticaria; therefore, stool test for $H$. Pylori antigen is recommended. ${ }^{42}$ Meta analysis showed that $H$. pylori might be associated with the occurrence 
and persistence of CSU. The effectiveness of $H$. pylori eradication therapy in suppressing CSU symptoms was significant. Interestingly, it was found that resolution of CSU was not associated with successful eradication of $H$. pylori infection. Patients diagnosed with urticaria who had undergone antibiotic therapy for $H$. pylori eradication showed significantly higher CSU remission, with or without $H$. pylori eradication. ${ }^{43}$

Autoimmune mechanisms are contributing to the pathogenesis of chronic urticaria; different pathogenic autoantibodies, causing a release of histamine after reaction with IgE epitopes, or with the a-chain of $\mathrm{Fc}$ epsilon $\mathrm{RI}$ receptors, are considered. ${ }^{44}$ Lesions can be as small as a few millimetres in diameter but can coalesce to form wheals as large as several centimetres wide. They often remit within 24 hours from time of onset. Urticaria may be accompanied by the presence of angioedema, which is a similar process that occurs at submucosal surfaces of the upper respiratory and gastrointestinal tracts and deeper layers of the skin including subcutaneous tissue. ${ }^{45}$

Urticaria is mainly classified based on clinical criteria: acute and chronic urticaria. Chronic urticaria comprises both CSU and chronic inducible urticaria that includes physical and non-physical urticarias. ${ }^{46} \mathrm{CSU}$ is a common and complex condition lasting for more than six weeks, and occurs without an identifiable causative factor. This skin disease in a subgroup of patients is related to autoreactive IgE, but the nature of this autoreactive IgE is still poorly characterised.

Formerly referred to as chronic idiopathic urticaria, CSU refers to recurrent urticaria lasting more than 6 weeks, that occurs in the absence of an identifiable trigger. Urticaria that are incited by a well-defined eliciting factor (e.g., pressure, temperature, vibration) are referred to as inducible urticaria and will not be further discussed in this review. Prevalence of chronic urticaria is estimated to be anywhere from $0.5-5 \%$ in the general population but is not truly known. ${ }^{47}$ Recent guidelines now include isolated idiopathic angioedema within the definition of CSU provided that other causes of angioedema, particularly those that are bradykinin mediated, have been excluded. ${ }^{48}$ Epidemiological data indicate that CSU in the general population is prevalent in approximately $1 \%^{49}$ and has a significant impact on quality of life. ${ }^{50}$

\section{CLINICAL APPROACHES}

\section{Diagnosis and Treatment Approaches in Chronic Spontaneous Urticaria Differ in Various Parts of the World}

There are no currently available biomarkers that can be used for the evaluation and management of patients diagnosed with CSU. Potential biomarkers of CSU severity and/or duration include basophil numbers and susceptibility to activation, inflammatory markers, markers of activation of the extrinsic coagulation pathway, immunoglobulin E, and vitamin D. Although the described markers are promising, further studies on representative and well-characterised patient populations are needed to determine the value of these clinical and biological markers for predicting the severity and course of disease in patients with $\mathrm{CSU} .^{51}$

An 'urticaria diary' can be very helpful for assessment. It should be used over several weeks to document information on the frequency and intensity of symptoms (e.g., wheals, itch, swelling, and systemic symptoms), possible relevance with physical factors, food intake and other activities (e.g., physical or emotional stress), and patient's medication.

The Urticaria Activity Score (UAS) is based on the evaluation of numbers of wheals and the intensity of itching using a O-3-point scale. It is calculated as the daily sum of the wheal and itch score, with a maximum score of six points per day and 42 points per week (for the UAS 7).

There are three possible underlying causes of CSU: infections, food intolerance, and autoreactivity. ${ }^{52}$ Bacterial infections, as well as viral, fungal, or parasitic infections can be a cause of CSU. The current guidelines recommend differential blood count analyses, determination of blood sedimentation rate and C-reactive protein, together with a focused patient history for discovering potentially relevant infections. ${ }^{2}$

Testing for $H$. pylori (stool, breath test, or demonstration of antigen/antibodies) are recommended. 
Higher levels of C-reactive protein (CRP) were associated with autologous serum skin test positivity, arterial hypertension, urticaria activity, quality of life impairment, inflammatory and coagulation markers, and poor response to antihistamines. Elevated levels of the sensitive inflammatory biomarker CRP are suggested for diagnosis and disease activity of CSU. ${ }^{53}$ Recent findings have demonstrated that IgE anti-thyroid antibodies are present at higher frequency and amounts in patients diagnosed with CSU and have greater potential to induce thyroid autoantibody-mediated skin reactions in these subjects versus healthy controls. ${ }^{54}$

A systematic review that assessed the relationship between vitamin D and CSU showed statistically significant lower serum vitamin $D$ levels in CSU-diagnosed patients compared to controls, and disease improvement after highdose vitamin D supplementation. Vitamin D deficiency was reported more commonly for patients diagnosed with CSU (34.3-89.7\%) than in controls (0.0-68.9\%). ${ }^{55}$ When history indicates a possible inducible pattern, physical skin tests (e.g., cold, heat, ultraviolet light, and pressure) as well as exercise tests should also be performed in order to verify or rule out inducible urticarias. To perform these tests antihistamines must be discontinued for at least 2-3 days.

If high or low temperature contact urticaria is suspected, skin testing with TempTest ${ }^{\circledast}$ (MOXIE $\mathrm{GmbH}$, Berlin, Germany) can be used as a diagnostic tool as well as to monitor responses to treatment. The provocation testing is performed for $5 \mathrm{~min}$ with a temperature $4-45^{\circ}$ on the volar forearm. The provocation time and temperature can be adapted individually. If a palpable, clearly visible wheal and flare-type skin reaction occurs, the test reaction is rated positive. Eosinopenia in patients diagnosed with CSU is associated with Type IIb autoimmunity (markers include autoantibodies, basophil tests, and/or autologous serum skin test), high disease activity, and poor response to treatment. Eosinophils should be explored as biomarkers and investigated for their contribution to the pathogenesis of CSU.

Subjects with IgE autoantibody-mediated CSU appear to have a faster onset of improvement in response to omalizumab than those with IgGmediated disease, due to the unique mechanisms by which this drug sequentially affects IgE levels and FceR1 status.
On the contrary, subjects who display a slow response to omalizumab are thought to have IgG antibodies against FceRI since down-regulation of this receptor occurs only after free IgE is first complexed by the drug. The authors validated this hypothesis by demonstrating a high correlation between length of time to the onset of omalizumab efficacy and positive basophil histamine release activity, with the latter predicting slower response times to treatment. 56

A recent study in 49 Caucasian patients diagnosed with CSU found elevated levels of specific IgE against a mix of Staphylococcus aureus enterotoxins in 51\% of patients compared to $33 \%$ in healthy controls. ${ }^{57}$ Total serum IgE levels and CSU disease activity were correlated with Staphylococcus enterotoxin B IgE levels. These results suggest a role of $S$. aureus enterotoxin IgE antibodies in the pathogenesis of CSU, in keeping with the current hypothesis of autoallergy being important in some patients.

In cholinergic urticaria that is actively (e.g., due to exercise) or passively (e.g., having a hot bath) induced, increases of the body temperature result in the appearance of itching and formation of wheals. Typically, the wheals are tiny, short-lived, and accompanied by a pronounced flare reaction, which is often localised on the limbs and trunk. ${ }^{58}$ This form of urticaria should be differentiated from exercise-induced urticaria/anaphylaxis, in which exercise but not passive warming provokes symptoms (cutaneous and more frequently than in cholinergic urticaria, systemic symptoms). In the differential diagnosis, attention should be given to food or drug-dependent exerciseinduced anaphylaxis.

\section{CONCLUSION}

The understanding, knowledge, and management of urticaria and angioedema are rapidly increasing. It was noted that in some patients, treatment of the underlying pathology led to clinical improvement. Further research is required to gain understanding of the mechanisms of interaction between infection and urticaria and angioedema. Interdisciplinary co-operation with dentists and ear, nose, and throat specialists, and X-ray and serological analysis for streptococcal (antistreptolysin) or staphylococcal infection should be performed to identify bacterial infections 
of the nasopharynx, e.g., recurrent sinusitis or tonsillitis. ${ }^{51,59}$

Data obtained indicated viral infection as a potential trigger and sometimes as the main aetiologic agent in causing acute or chronic urticaria. In every case, urticarial manifestation cleared up after either healing or controlling of the viral infection. However, prospective studies and well-structured research are needed to better clarify the role of viruses in the pathogenesis of urticaria and their relative prevalence. ${ }^{60}$

The author can hypothesise that the underlying lasting immune response to an infection, rather than infection itself, is the causative factor for persistence of urticaria, alongside autoimmune factors. Antibodies and co-factors acting together reduce the threshold of reactivity leading to symptoms.

Many questions and unmet needs remain to be addressed, such as the development of routine diagnostic tests for autoimmune urticaria and angioedema; the global dissemination and consistent use of tools to assess disease activity, impact, and control; and the development of more effective and well-tolerated longterm treatments for all forms of urticaria and angioedema.

\section{References}

1. Maurer M et al. Mast cells: promoters of health and modulators of disease. J Allergy Clin Immunol. 2019;144(4S):S1-S3.

2. Zuberbier $T$ et al. The EAACl/GA(2) LEN/EDF/WAO guideline for the definition, classification, diagnosis and management of urticaria. The 2017 revision and update. Allergy. 2018;73(7):1393-414.

3. Poonawalla T, Kelly B. Urticaria: a review. Am J Clin Dermatol. 2009;10(1):9-21.

4. Weller K et al. Chapter 29: Chronic Urticaria. Evidence-Based Dermatology. 2014;3:210-22.

5. Kanani A et al. Urticaria and angioedema. Allergy Asthma Clin Immunol. 2018;14(Suppl 2):59.

6. Minciullo PL et al. Urticaria and bacterial infections. Allergy Asthma Proc. 2014;35(4):295-302.

7. Sackesen $\mathrm{C}$ et al. The etiology of different forms of urticaria in childhood. Pediatr Dermatol. 2004;21(2):102-8.

8. Rosenblum MD et al. Mechanisms of human autoimmunity. J Clin Invest. 2015;125(6):2228-33.

9. Puxeddu I et al. Mediators of inflammation and angiogenesis in chronic spontaneous urticaria: are they potential biomarkers of the disease?. Mediators Inflamm. 2017;2017:4123694.

10. Radonjic-Hoesli, S et al. Urticaria and angioedema: an update on classification and pathogenesis. Clinic Rev Allerg Immunol. 2018;54(1):88101.

11. Kolkhir P et al. Autoimmune chronic spontaneous urticaria: what we know and what we do not know. J Allergy Clin Immunol. 2017;139(6):1772-81.e1.
12. Vasagar $\mathrm{K}$ et al. Evidence of in vivo basophil activation in chronic idiopathic urticaria. Clin Exp Allergy. 2006;36(6):770-6

13. Maurer $M$ et al. Immunoglobulin E-mediated autoimmunity. Front Immunol. 2018;9:689.

14. Ulambayar B et al. Detection of circulating IgG autoantibody to FceRla in sera from chronic spontaneous urticaria patients. $\mathrm{J}$ Microbiol Immunol Infect. 2020;53(1):141-7.

15. Gaig P et al. Epidemiology of urticaria in Spain. J Investig Allergol Clin Immunol. 2004;14(3):214-20.

16. Bernstein JA et al. The diagnosis and management of acute and chronic urticaria: 2014 update. J Allergy Clin Immunol. 2014;133(5):1270-7.

17. Maurer M et al. Relevance of food allergies and intolerance reactions as causes of urticaria. Hautarzt. 2003;54(2):138-43.

18. Napolitano $M$ et al. Salt-dependent aquagenic urticaria in children: report of two cases. Pediatr Allergy Immunol. 2018;29(3):324-6.

19. Napolitano M, Patruno C. Chronic urticaria can be caused by cancer and resolves with its cure. Allergy. 2018;73(8):1750-1.

20. Larenas-Linnemann D et al. Very rarely chronic urticaria can be caused by cancer and if so, resolves with its cure. Allergy. 2018;73(9):1925-6.

21. Yon DK, Cho YS, Ha EK, Jee HM, Song JY, Jung $Y H$, et al. Exposure to phthalates is associated with acute urticaria in children. Pediatr Allergy Immunol. 2018;29(6):657-60.

22. Kulthanan $\mathrm{K}$ et al. Acute urticaria: etiologies, clinical course and quality of life. Asian Pac J Allergy Immunol.
2008;26(1):1-9.

23. Wu CC et al. Association of acute urticaria with mycoplasma pneumoniae infection in hospitalized children. Ann Allergy Asthma Immunol. 2009;103(2):134-9.

24. McMahon AW et al. Inactivated influenza vaccine (IIV) in children $<2$ years of age: examination of selected adverse events reported to the Vaccine Adverse Event Reporting System (VAERS) after thimerosal-free or thimerosal-containing vaccine. Vaccine. 2007;26(3):427-9.

25. Lipsker D, Boeckler P. Acute urticaria and dry cough with interstitial pneumonia: a clue for the diagnosis of primary parvovirus B19 infection. Clin Exp Dermatol. 2006;31(3):473-4.

26. Zuberbier T. Urticaria. Allergy. 2003;58(12):1224-34.

27. Schuller DE, Elvey SM. Acute urticaria associated with streptococcal infection. Pe-diatrics. 1980;65(3):5926.

28. Scully LJ, Ryan AE. Urticaria and acute hepatitis $A$ virus infection. Am J Gastroen-terol. 1993;88(2):277-8.

29. Frick OL et al. Development of allergy in children: I. Association with virus infec-tions. J Allergy Clin Immunol. 1979;63(4):228-41.

30. Dienstag JL et al. Urticaria associated with acute viral hepatitis type B: studies of pathogenesis. Ann Intern Med. 1978;89(1):34-40.

31. Cherry JD et al. Coxsackie A9 infections with exanthemas, with particular reference to urticaria. Pediatrics. 1963;31:819-823.

32. $\mathrm{Gu} \mathrm{H}$ et al. Association between helicobacter pylori infection and chronic urticaria: a metaanalysis. Gastroenterol Res Pract. 


\section{5;2015:486974.}

33. Vaida GA et al. Testing for hepatitis B virus in patients with chronic urticaria and an-gioedema. J Allergy Clin Immunol. 1983;72(2):193-8

34. Patella $\vee$ et al. Protein Fv produced during vital hepatitis is a novel activator of human basophils and mast cells. J Immunol. 1993;151(10):5685-98.

35. Genovese A et al. Protein Fv produced during viral hepatitis is an endogenous immunoglobulin superantigen activating human heart mast cells. Int Arch Allergy Im-munol. 2003:132(4):336-45

36. Kolkhir, $\mathrm{P}$ et al. Comorbidity of viral hepatitis and chronic spontaneous urticaria: a systematic review. Allergy. 2018;73(10):1946-53

37. Dreyfus DH. Serological evidence that activation of ubiquitous human herpesvirus-6 (HHV-6) plays a role in chronic idiopathic/spontaneous urticaria (CIU). Clin Exp Immunol. 2016;183(2):230-8.

38. Zhang, JJ et al. Clinical characteristics of 140 patients infected with SARSCoV-2 in Wuhan, China. Allergy. 2020;75(7):1730-41.

39. Recalcati S. Cutaneous manifestations in COVID-19: a first perspective. $\mathrm{J}$ Eur Acad Dermatol Venereol. 2020;34(5):e212-3.

40. Sachdeva $M$ et al. Cutaneous manifestations of COVID-19: report of three cases and a review of literature. J Dermatol Sci. 2020;98(2):75-81.

41. Tüzün $Y$ et al. The role of Helicobacter pylori infection in skin diseases: facts and controversies. Clin Dermatol. 2010:28(5):478-82
42. Shabrawy RM, Gharib K. Helicobacter pylori infection as a risk factor in patients suf-fering from food allergy and urticaria. Egypt J Immunol. 2016;23(1):67-75

43. Kim HJ et al. Systematic review and meta-analysis: effect of Helicobacter pylori eradication on chronic spontaneous urticaria. Helicobacter. 2019:24(6):e12661.

44. Zuberbier $T$ et al. EAACI/GA(2)LEN/ EDF/WAO guideline: management of urticar-ia. Allergy. 2009;64(10):142743

45. Haynes B et al, "The Immune System in Health and Disease", Harrison's Principles of Internal Medicine, volume 2 (2015) 19th edition, McGraw Hill Education.

46. Maurer $M$ et al. Unmet clinical needs in chronic spontaneous urticaria. A GA(2)LEN task force report. Allergy. 2011;66(3):317-30.

47. Lapi $\mathrm{F}$ et al. Epidemiology of chronic spontaneous urticaria: results from a nation-wide, populationbased study in Italy. $\mathrm{Br} \mathrm{J}$ Dermatol. 2016:174(5):996-1004.

48. Maurer $\mathrm{M}$ et al. Definition, aims, and implementation of GA2 LEN/HAEi angioedema centers of reference and excellence. Allergy. 2020;75(8):211523.

49. O'Donnell BF et al. The impact of chronic urticaria on the quality of life. Br J Dermatol. 1997;136(2):197-201.

50. Asero $\mathrm{R}$ et al. Chronic urticaria: focus on pathogenesis. 2017;6:1095.

51. Sánchez-Borges $M$ et al. Factors linked to disease severity and time to remission in patients with chronic spontaneous urticaria. J Eur Acad
Dermatol Venereol. 2017:31(6):964-71.

52. Maurer M, Grabbe J. Urticaria: its history-based diagnosis and etiologically oriented treatment. Dtsch Arztebl Int. 2008;105(25):45865.

53. Kolkhir P et al. C-reactive protein is linked to disease activity, impact, and response to treatment in patients with chronic spontaneous urticaria. Allergy. 2018;73(4):940-8.

54. Sánchez J et al. Causal relationship between anti-TPO IgE and chronic urticaria by in vitro and in vivo tests. Allergy Asthma Immunol Res. 2019;11(1):29-42.

55. Tuchinda $\mathrm{P}$ et al. Relationship between vitamin $\mathrm{D}$ and chronic spontaneous urticaria: a systematic review. Clin Transl Allergy. 2018;8:51.

56. Gericke J et al. Serum autoreactivity predicts time to response to omalizumab therapy in chronic spontaneous urticaria. J Allergy Clin Immunol. 2017:139(3):1059-61.

57. Altrichter $\mathrm{S}$ et al. In chronic spontaneous urticaria, IgE against staphylococcal enterotoxins is common and functional. Allergy. 2018:73(7):1497-504

58. Beck $L A$ et al. A review of international recommendations for the diagnosis and management of chronic Urticaria. Acta Derm Venereol. 2017;97(2):149-58.

59. Wedi B et al. Infections and chronic spontaneous urticaria. A review. Hautarzt. 2010;61(9):758-64.

60. Imbalzano E et al. Association between urticaria and virus infections: a systematic review. Allergy Asthma Proc. 2016:37(1):18-22. 